\title{
STELLAR BINARY COMPANIONS TO SUPERNOVA PROGENITORS
}

\author{
CHRISTOPHER S. KOCHANEK ${ }^{1,2}$ \\ ${ }^{1}$ Department of Astronomy, The Ohio State University, 140 West 18th Avenue, Columbus, OH 43210, USA \\ ${ }^{2}$ Center for Cosmology and AstroParticle Physics, The Ohio State University, 191 W. Woodruff Avenue, Columbus, OH 43210, USA \\ Received 2009 September 23; accepted 2009 November 3; published 2009 December 7
}

\begin{abstract}
For typical models of binary statistics, $50 \%-80 \%$ of core-collapse supernova (ccSN) progenitors are members of a stellar binary at the time of the explosion. Independent of any consequences of mass transfer, this has observational consequences that can be used to study the binary properties of massive stars. In particular, the secondary companion to the progenitor of a Type $\mathrm{Ib} / \mathrm{c} \mathrm{SN}$ is frequently $(\sim 50 \%)$ the more optically luminous star since the high effective temperatures of the stripped progenitors make it relatively easy for a lower luminosity, cooler secondary to emit more optical light. Secondaries to the lower mass progenitors of Type II SN will frequently produce excess blue emission relative to the spectral energy distribution of the red primary. Available data constrain the models weakly. Any detected secondaries also provide an independent lower bound on the progenitor mass and, for historical SN, show that it was not a Type Ia event. Bright ccSN secondaries have an unambiguous, post-explosion observational signature - strong, blueshifted, relatively broad absorption lines created by the developing SN remnant (SNR). These can be used to locate historical SN with bright secondaries, confirm that a source is a secondary, and, potentially, measure abundances of ccSN ejecta. Luminous, hot secondaries will re-ionize the SNR on timescales of 100-1000 yr that are faster than re-ionization by the reverse shock, creating peculiar $\mathrm{H}_{\text {II }}$ regions due to the high metallicity and velocities of the ejecta.
\end{abstract}

Key words: stars: evolution - supergiants - supernovae: general

\section{INTRODUCTION}

The discovery of the blue progenitor to SN 1987A (Gilmozzi et al. 1987) triggered interest in the binarity of core-collapse supernova ( $\mathrm{ccSN})$ progenitors, since mass transfer provided a simple explanation for how a relatively low-mass $\sim 15-20 M_{\odot}$ star could explode as a blue supergiant (Podsiadlowski \& Joss 1989; de Loore \& Vanbeveren 1992). Later population studies showed that $\sim 25 \%$ of massive stars may undergo sufficient mass transfer to transform a primary that would explode as a red supergiant in a Type II SN into a stripped Helium (or beyond) star that would explode as a Type Ib/c SN (e.g., Podsiadlowski et al. 1992; Eldridge et al. 2008). If the secondary gains enough mass as part of this process, its evolution accelerates and it can explode as a Type II SN while still a blue supergiant.

Many aspects of these possibilities were confirmed by the Type IIb SN 1993J in M81 (3.6 Mpc). A binary companion was first suggested based on excess blue emission in the spectral energy distribution (SED) of the progenitor (Aldering et al. 1994), and later confirmed in post-explosion imaging and spectroscopic observations (Maund et al. 2004; Maund \& Smartt 2009). Binary evolution models produce SN 1993J starting with two similar mass stars (Podsiadlowski et al. 1993; Maund et al. 2004; Stancliffe \& Eldridge 2009). Mass transfer removes almost all the Hydrogen envelope of the primary prior to the explosion, leading to the Type IIb transition from a Type II to a Type Ib spectral type as the $\mathrm{SN}$ evolves.

While direct searches for SN progenitors are now common, direct constraints on binary companions are relatively rare. Smartt (2009) reviews the status of progenitor searches (e.g., Li et al. 2007; Gal-Yam et al. 2007; Smartt et al. 2009). For Type IIP SN there are 20 events with adequate pre-SN data, with six detections and 12 strong upper bounds. These progenitors are red supergiants with $7 M_{\odot} \lesssim M_{p} \lesssim 17 M_{\odot}$, which is surprising given that locally red supergiants are found up to masses of
$25 M_{\odot}$ (Levesque et al. 2005). No progenitor of a Type Ib/c SN has been identified out of 10 cases with adequate pre-explosion data, which may be further evidence that mass transfer is playing an important role in creating these $\mathrm{SNe}$ (Smartt 2009).

Because the data on SN progenitors rarely have broad spectral coverage and there is little adequate post-outburst data, there are only a handful of constraints on companion stars other than those for SN 1993J. Two additional Type IIb SN, SN 2008ax (Crockett et al. 2008) and SN 2001ig (Ryder et al. 2006), show evidence for a secondary. For SN 2008ax, there is a blue excess to the SED of the progenitor, while for SN 2001ig there is a candidate for the surviving, blue secondary in post-explosion images. The existence of a companion is limited to $M_{s} \lesssim 20 M_{\odot}$ for the Type Ic SN 2002ap (Crockett et al. 2007), which also has the tightest limits on the progenitor of any Type $\mathrm{Ib} / \mathrm{c} \mathrm{SN}$, to $L \lesssim 2 L_{\odot}$ for SN 1987A (Graves et al. 2005), and to roughly $M_{V} \gtrsim 2$ mag for the Type IIb SN Cassiopeia A (Fesen et al. 2006; Krause et al. 2008). Finally, the Type IIP SN 2008bk (Mattila et al. 2008) and SN 2005cs (Maund et al. 2005; Li et al. 2006) have upper bounds on excesses to their SEDs at $V$ band of approximately $1 \mathrm{mag}$. That the constraints are few is not surprising, given that it will generally be more challenging to identify the secondaries. We should note that there is also indirect evidence for the presence of a binary companion from the structure of some radio supernova light curves (see Van Dyk 2004).

Five years ago, limits and detections of SN progenitors would have been equally limited, but the passage of time renders all studies of SN progenitors and their secondaries easier, since both the number of nearby SN and the amount of useful archival data continuously increase. This suggests that an examination of our expectations for the properties of binary companions to ccSN progenitors and of any strategies for detection are worthwhile. In this paper, we first estimate the fraction of ccSN that have a stellar binary companion at the time of the $\mathrm{SN}$ in Section 2 and then examine the optical properties of these companions in 
Section 3. In Section 4, we examine whether these models are constrained by any of the existing data on SN progenitors and their companions. In Section 5, we point out that there is a simple technique to discover and unambiguously confirm the brighter secondary companions to ccSN even when the position of the ccSN is poorly constrained. Finally, in Section 6, we summarize the results and discuss the observational prospects. We examine these questions in the "wide binary" limit, where there have been no interactions between the two stars. In most cases, adding the interactions will enhance the visibility of the secondary, as mass transfer frequently leads to a hotter primary radiating less in the optical and a more massive and luminous secondary. We will consider these effects in C. S. Kochanek et al. (2010, in preparation). In the Appendix, we discuss other possibilities for locating historical supernovae sufficiently accurately to search for surviving secondaries.

\section{THE FRACTION OF ccSN IN STELLAR BINARIES}

We assume that the distribution of primary masses $M_{p}$ is Salpeter, $d N / d M_{p} \propto M_{p}^{-x}$ with $x=2.35$, and that fraction $F$ of the primaries have binary companions distributed in mass $M_{s}$ as $f(q)$ with $q_{\min } \leqslant q=M_{s} / M_{p} \leqslant q_{\max }$ where $\int d q f(q) \equiv 1$. With these definitions, the joint distribution of primary and secondary stars in mass is

$$
\frac{d N}{d M_{p} d M_{s}}=F M_{p}^{-x-1} f(q),
$$

and the distribution of secondary masses is

$$
\frac{d N}{d M_{s}}=F f_{q} M_{s}^{-x}
$$

where

$$
f_{q}=\int_{q_{\min }}^{q_{\max }} q^{x-1} f(q) d q .
$$

Note that the distribution of secondaries in mass is also Salpeter in form, and that at a fixed stellar mass there are $F f_{q}$ secondaries for every primary. The fraction at fixed mass $F f_{q}$ is smaller than the global fraction $F$ because secondaries of a given mass are associated with rarer, higher mass primaries. When we observe a ccSN from a star of given mass, it can be the collapse of a single star that was never in a binary, the primary of a binary or the secondary of a binary, and the relative probabilities of these three cases are

$$
f_{\text {single }}=\frac{1-F}{1+F f_{q}}, \quad f_{p}=\frac{F}{1+F f_{q}}, \quad \text { and } \quad f_{s}=\frac{F f_{q}}{1+F f_{q}} .
$$

As long as the primary mass function is a power law and the secondary mass function depends only on $q$, these fractions are mass independent. The primary stars for the fraction $f_{s}$ that are exploding secondaries have already collapsed, so the secondary is either no longer in a binary (because it was disrupted by the mass ejection and/or velocity kicks from the explosion of the primary) or the primary is now a stellar remnant. In either case, there is no observable stellar companion. Thus, the fraction of ccSN in stellar binaries is simply $f_{p}$.

We consider three models for the distribution of secondaries. The first two are based on Kobulnicky \& Fryer (2007) and are limited to $0.02<q<1$. The "low-mass" model, $f(q) \propto q^{-0.6}$, is biased to low-mass binary companions, while the "uniform" model with $f(q)$ constant gives equal weight to all masses.
Table 1

Binary Status at the Time of ccSN

\begin{tabular}{ccccc}
\hline \hline Model & $F$ & $f_{\text {single }}$ & $f_{p}$ & $f_{s}$ \\
\hline Low mass & 1.00 & 0.00 & 0.78 & 0.22 \\
& 0.75 & 0.21 & 0.62 & 0.18 \\
& 0.50 & 0.44 & 0.44 & 0.13 \\
Uniform & 0.25 & 0.70 & 0.23 & 0.07 \\
& 1.00 & 0.00 & 0.70 & 0.30 \\
& 0.75 & 0.19 & 0.57 & 0.25 \\
& 0.50 & 0.41 & 0.41 & 0.18 \\
Twins & 0.25 & 0.67 & 0.23 & 0.10 \\
& 1.00 & 0.00 & 0.60 & 0.40 \\
& 0.75 & 0.17 & 0.50 & 0.33 \\
& 0.50 & 0.38 & 0.38 & 0.25 \\
& 0.25 & 0.64 & 0.21 & 0.14 \\
\hline
\end{tabular}

Notes. The model column defines the $f(q)$ distribution of the secondary stars (see the text), $F$ is the fraction of primaries that are in binaries, and $f_{\text {single }}, f_{p}$, and $f_{s}$ are the fraction of ccSN that are isolated stars, binary primaries, and binary secondaries, respectively.

The third, "twins" model is based on Pinsonneault \& Stanek (2006) and puts half of the companions in a twin distribution with $0.9<q<1$ and the other half with $0.02<q<0.9$, using uniform distributions for each mass ratio interval. The fractions of stars that are secondaries at a fixed stellar mass if $F=1$ are $f_{q}=0.288, f_{q}=0.434$, and $f_{q}=0.644$ for the low-mass, uniform and twins models, respectively. Table 1 summarizes the fractions (Equation (4)) of ccSN that will be isolated stars, binary primaries, and binary secondaries for each of these models and binary fractions of $F=1,3 / 4,1 / 2$, and $1 / 4$. Biasing the binary distribution to lower masses increases $f_{p}$ because there are fewer, diluting massive secondaries. Reducing the binary fraction $F$, particularly for high binary fractions, has a weaker effect on $f_{p}$, because the change is partly compensated by the reduced numbers of exploding secondaries. If the binary fraction $F \gtrsim 0.7$, as favored by Kobulnicky \& Fryer (2007), then $50 \%-80 \%$ of ccSN are part of a stellar binary at the time of the explosion.

\section{OBSERVABILITY}

That companions are common does not imply that they are observable, so we next make a simple model of their visibility. We took the Marigo et al. (2008) solar metallicity isochrones, called the most massive star along the isochrone the SN progenitor, and then selected secondaries from the less massive stars along the same isochrone. Massive stars reach the main sequence rapidly compared to their post-main-sequence life times (e.g., Zinnecker \& Yorke 2007), so we can ignore small differences in the pre-main sequence evolution of the binary stars and simply start from the zero-age main sequence (ZAMS). We used the estimated Hubble Space Telescope (HST)/ACS $H S T / \mathrm{ACS} B(\mathrm{~F} 435 \mathrm{~W}), V(\mathrm{~F} 555 \mathrm{~W})$, and $I(\mathrm{~F} 814 \mathrm{~W})$ magnitudes. We use the same binary distributions as in Section 2 and assume $F=1$. The resulting distributions can be scaled to lower binary fractions using Table 1.

We also examined the Geneva (Lejeune \& Schaerer 2001) and Cambridge STARS (Eldridge et al. 2008) model sequences, but were able to use them only to spot check the results. Lejeune $\&$ Schaerer (2001) provide end-phase optical magnitudes only for $M \lesssim 25 M_{\odot}$, while Eldridge et al. (2008) provide histories for discrete masses that are too coarsely sampled to turn into isochrones. Where we can compare results between Marigo 


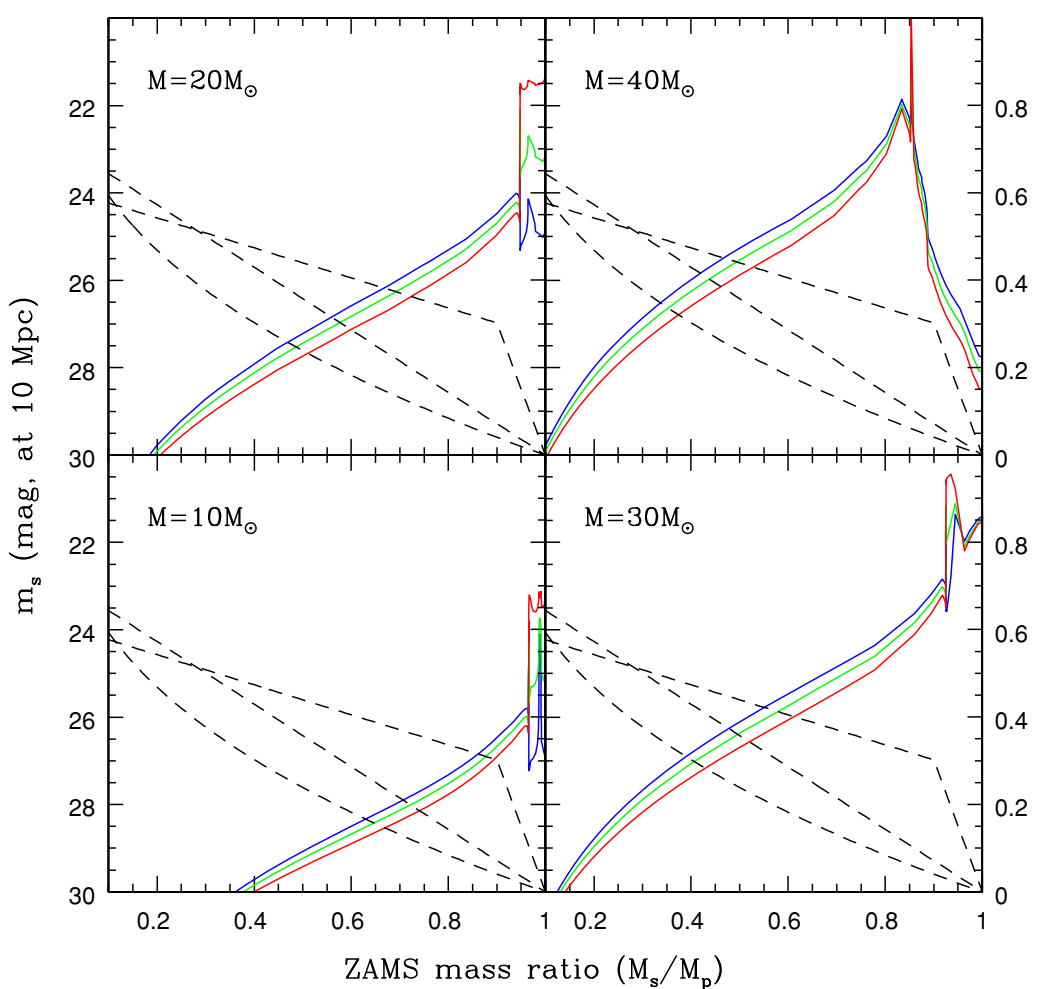

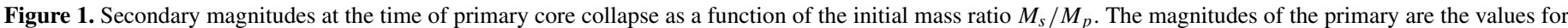

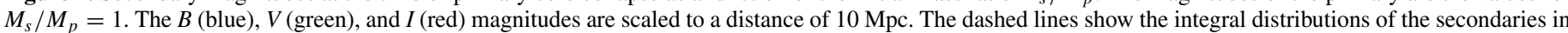

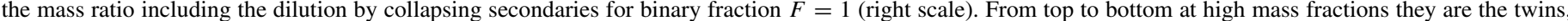

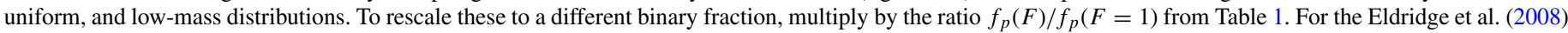
evolution models, the $30 M_{\odot}$ case would resemble the $40 M_{\odot}$ case.

et al. (2008) and Lejeune \& Schaerer (2001), they are similar. The final phases of the Marigo et al. (2008) and Eldridge et al. (2008) models are generally similar over the mass range $10 M_{\odot} \lesssim M \lesssim 50 M_{\odot}$, but with significant differences in the onset of extreme mass loss. In the Eldridge et al. (2008) models, the transition occurs abruptly at $M \simeq 28 M_{\odot}$, while in the Marigo et al. (2008) models there is a steady shift over the range from $20 M_{\odot}<M<40 M_{\odot}$. Thus, the $M_{p}=30 M_{\odot}$ case we construct from Marigo et al. (2008) resembles the $M_{p}=20 M_{\odot}$ case, while in the Eldridge et al. (2008) models it would resemble the $M_{p}=40 M_{\odot}$ case.

Figure 1 shows the distributions of secondaries in mass and magnitude relative to primaries of fixed mass, along with the integral distribution of secondaries in mass for the $F=1$ models, including the dilution by the ccSN of secondaries. To scale these fractions to a different $F$, simply multiply by the ratio $f_{p}(F) / f_{p}(F=1)$ from Table 1 . We show the distributions for primary masses of $M_{p} \simeq 10,20,30$, and $40 M_{\odot}$ and scale the magnitudes to a distance of $10 \mathrm{Mpc}$. For the lower mass cases, the stars are brightest just when they collapse, and only a narrow range of secondary masses can approach the luminosity of the primary. Most secondaries are fainter, blue main sequence stars. The secondaries of more massive stars are more visible for three reasons. First, for the same mass ratio they are simply more luminous. Second, the luminosity differences between main sequence and evolved stars of similar mass are smaller. Finally, mass loss leads to primary effective temperatures too high to efficiently radiate in the optical. For the Marigo et al. (2008) models we see this only for the $M_{p}=40 M_{\odot}$ case, but in the Eldridge et al. (2008) models the $M_{p}=30 M_{\odot}$ case would show the same properties.
In Figure 2, we combine the distributions in secondary mass and luminosity to examine the distribution of secondary magnitudes relative to their primaries. The $M_{p}=10$ and $20 M_{\odot}$ primaries undergo core collapse as red stars close to the peak optical luminosity of all stars on the isochrone and generally have a blue companion. As a result, it is difficult for the companions to significantly perturb the combined SED that would be measured pre-explosion other than by creating a blue excess. For the $M_{p}=40 M_{\odot}$ case, the secondaries are frequently brighter than the primary for the reasons discussed above. In general, however, both stars are blue, so the overall SED will not be strongly distorted by the combination. While many of the secondaries are also massive stars, most are relatively faint and can only be found directly in relatively nearby galaxies $(<10 \mathrm{Mpc})$.

Table 2 summarizes several of these observational points for $F=1$. We first examined the contribution of the secondary to the blue flux of the combined SED by computing the fraction of ccSN where a secondary contributes more than $10 \%$ or $100 \%$ of the blue flux of the primary. For lower mass stars, where the primary explodes as a red star, the secondary generally distorts the SED and appears as a blue excess. For the low-mass and uniform secondary distributions, the secondary is rarely (5\%$15 \%)$ as bright in the blue as the primary, but frequently $(25 \%-$ $50 \%)$ a significant $(>10 \%)$ perturbation. Spectral distortions are far more common in the twins model. Contamination at redder wavelengths for these lower mass stars is minimal and will have no effect on the inferences about Type IIP progenitors by Smartt et al. (2009). For the high-mass $M_{p}=40 M_{\odot}$ case, the secondary is frequently brighter than the primary at all optical wavelengths because both stars are hot enough for their optical 


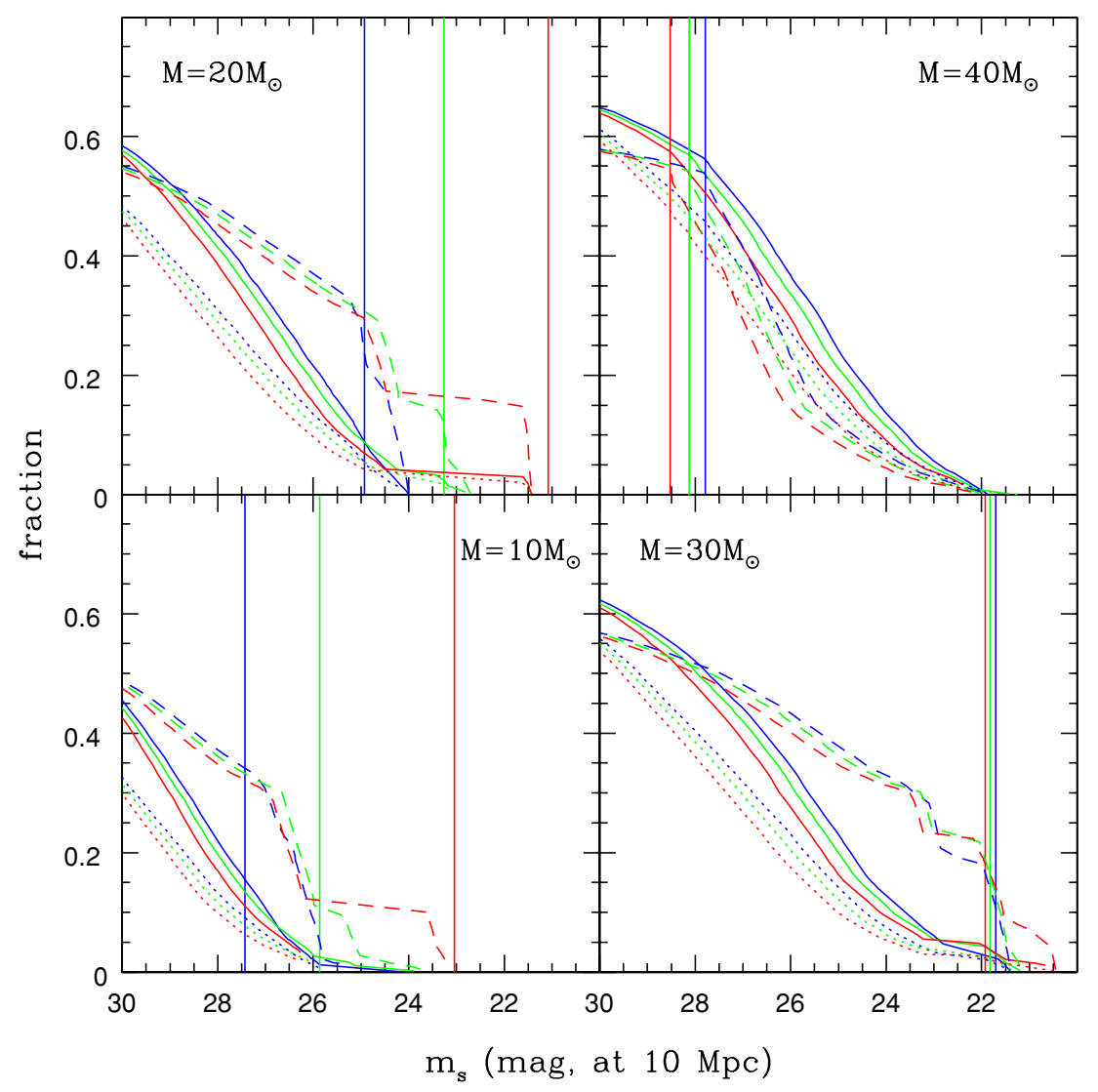

Figure 2. Integral distributions of companion magnitudes in the $B$ (blue), $V$ (green), and $I$ (red) bands at a distance of 10 Mpc. The low-mass (dotted), uniform (solid), and twins (dashed) distributions are shown for a binary fraction $F=1$ and the diluting effects of collapsing secondaries are included. To rescale these to a different binary fraction, multiply by the ratio $f_{p}(F) / f_{p}(F=1)$ from Table 1 . The vertical lines mark the magnitudes of the primary at the time of collapse. Note how the intrinsically most luminous $M_{p}=40 M_{\odot}$ progenitor is optically fainter than the three lower mass progenitors. For the Eldridge et al. (2008) evolution models, the $30 M_{\odot}$ case would resemble the $40 M_{\odot}$ case.

colors to be saturated. The intermediate $M_{p}=30 M_{\odot}$ case is the least affected by the presence of a secondary in the Marigo et al. (2008) models because the primary is both intrinsically luminous and has retained a significant Hydrogen envelope. Nonetheless, many of the secondaries are intrinsically luminous and relatively easy to detect after the explosion fades.

We can also use these models to roughly estimate the fraction of systems which will interact. From the Marigo et al. (2008) models, we can estimate the initial and maximum radius of the stars. We distribute the semimajor axes $a$ uniformly in $\log a$ (Kobulnicky \& Fryer 2007) from a minimum radius $a_{\min }$ to $10^{5} a_{\min }$ where the minimum radius was set so that the initial stellar radii were $1 / 2$ the initial Roche lobe radius, following Belczynski et al. (2008). With these assumptions, 25\%-33\% of the primaries will fill their Roche lobes at their maximum stellar radii. The fraction depends weakly on the dynamic range chosen for the semimajor axes, rising to $30 \%-45 \%$ if we reduce it by an order of magnitude, and dropping to $20 \%-30 \%$ if we raise it by an order of magnitude. These ranges are consistent with more detailed studies (e.g., Podsiadlowski et al. 1992; Kobulnicky \& Fryer 2007; Eldridge et al. 2008), and should mean that adding interactions changes the details of our picture but not the broad outline.

\section{CONSTRAINTS}

Most accountings of the role of binaries in $\mathrm{SN}$ are based on the distribution of supernova types (e.g., Podsiadlowski et al.
1992; Kobulnicky \& Fryer 2007; Eldridge et al. 2008). Suppose stars from $M_{\min }<M<M_{\text {strip }}$ explode as non-stripped Type II SN (IIP, IIL, IIn), and stars with $M>M_{\text {strip }}$ explode as stripped Type $\mathrm{Ilb}$ and $\mathrm{Ib} / \mathrm{c} \mathrm{SN}$ where the fraction of stripped $\mathrm{SN}$ is about $f_{\text {strip }} \simeq 35 \%$ and $M_{\text {min }} \simeq 8 M_{\odot}$ (Smartt et al. 2009). In the absence of any binary interactions and assuming a Salpeter mass function, producing the stripped $\mathrm{SN}$ requires the stripping mass scale to be $M_{\text {strip }}=f_{\text {strip }}^{-0.74} M_{\min } \simeq 2.2 M_{\min } \simeq 18 M_{\odot}$. This is much lower than the mass scale $M \simeq 25 M_{\odot}$ beyond which models for massive stars lose their envelopes prior to core collapse (Lejeune \& Schaerer 2001; Eldridge et al. 2008; Marigo et al. 2008), although it is curiously similar to the upper bound on Type IIP progenitors found by Smartt et al. (2009). If we now allow fraction $f_{i}$ of the progenitors with $M<M_{\text {strip }}$ to interact and become stripped supernovae by mass transfer processes, then the interacting fraction must be $f_{i}=0.08,0.17$, and 0.22 for $M_{\text {strip }}=20,25$, and $30 M_{\odot}$ in order to produce the observed numbers of stripped $\mathrm{SN}$, and the fractions of stripped SN created by binary interactions are $16 \%, 38 \%$, and $52 \%$. While this is overly simplified, it is roughly consistent with the detailed models of binary evolution, and, if only $\sim 25 \%$ of binaries are interacting, it requires the overall binary fraction $F$ to be high, consistent with Kobulnicky \& Fryer (2007). Note, however, that creating too large a fraction of stripped SN by binary interactions of lower mass stars may by inconsistent with the strong positional correlations between Type $\mathrm{Ib} / \mathrm{c} \mathrm{SN}$ and $\mathrm{H} \alpha$ emission from young massive stars (Anderson \& James 2008). Stripped ccSN due to binary evolution are likely to 
Table 2

Observability of Binary Companions

\begin{tabular}{|c|c|c|c|c|c|c|c|c|c|}
\hline \multirow[t]{2}{*}{ Model } & \multirow{2}{*}{$\begin{array}{c}M_{p} \\
\left(M_{\odot}\right)\end{array}$} & \multicolumn{2}{|c|}{$B$ Band } & \multicolumn{2}{|c|}{ I Band } & \multirow{2}{*}{$\begin{array}{c}I_{s} \leqslant 26 \\
(10 \mathrm{Mpc})\end{array}$} & \multirow{2}{*}{$\begin{array}{l}I_{s} \leqslant 26 \\
(3 \mathrm{Mpc})\end{array}$} & \multirow{2}{*}{$\begin{array}{c}I_{s} \leqslant 24 \\
(10 \mathrm{Mpc})\end{array}$} & \multirow{2}{*}{$\begin{array}{l}I_{s} \leqslant 24 \\
(3 \mathrm{Mpc})\end{array}$} \\
\hline & & $>10 \%$ & $>100 \%$ & $>10 \%$ & $>100 \%$ & & & & \\
\hline \multirow[t]{4}{*}{ Low mass } & 10 & 0.32 & 0.09 & 0.02 & 0.00 & 0.02 & 0.15 & 0.02 & 0.03 \\
\hline & 20 & 0.26 & 0.05 & 0.02 & 0.00 & 0.09 & 0.33 & 0.02 & 0.14 \\
\hline & 30 & 0.09 & 0.02 & 0.07 & 0.02 & 0.17 & 0.42 & 0.06 & 0.23 \\
\hline & 40 & 0.63 & 0.46 & 0.65 & 0.47 & 0.21 & 0.48 & 0.06 & 0.28 \\
\hline \multirow[t]{4}{*}{ Uniform } & 10 & 0.45 & 0.15 & 0.02 & 0.00 & 0.02 & 0.25 & 0.02 & 0.05 \\
\hline & 20 & 0.38 & 0.09 & 0.04 & 0.00 & 0.16 & 0.45 & 0.04 & 0.22 \\
\hline & 30 & 0.14 & 0.03 & 0.12 & 0.04 & 0.28 & 0.54 & 0.09 & 0.35 \\
\hline & 40 & 0.65 & 0.56 & 0.66 & 0.57 & 0.29 & 0.58 & 0.09 & 0.37 \\
\hline \multirow[t]{4}{*}{ Twins } & 10 & 0.48 & 0.34 & 0.10 & 0.00 & 0.10 & 0.38 & 0.10 & 0.22 \\
\hline & 20 & 0.45 & 0.24 & 0.16 & 0.00 & 0.34 & 0.48 & 0.16 & 0.37 \\
\hline & 30 & 0.34 & 0.12 & 0.33 & 0.19 & 0.40 & 0.52 & 0.32 & 0.44 \\
\hline & 40 & 0.58 & 0.54 & 0.59 & 0.54 & 0.14 & 0.55 & 0.05 & 0.23 \\
\hline
\end{tabular}

Notes. The model column defines the $f(q)$ distribution of the secondary stars (see the text), $M_{p}$ is the primary mass, and the $B$ band and $I$ band columns give the fraction of secondaries exceeding $10 \%$ and $100 \%$ of the primary's flux in that band. The remaining columns show the fraction of SN with secondaries brighter than either $I=26$ or 24 mag at either $10 \mathrm{Mpc}$ or $3 \mathrm{Mpc}$. These are calculated for $F=1$ but can be corrected to lower values of $F$ using Table 1.

show the weaker positional correlations of Type II SN with $\mathrm{H} \alpha$ emission.

The failure to detect Type Ib/c "progenitors" provides our first constraint. Suppose our $M_{p}=40 M_{\odot}$ model is typical for massive stars stripped by winds to become Type Ib/c SN, so that the progenitor detection limits are also strong limits on secondaries. Based on Smartt's (2009) upper limits on 10 Type $\mathrm{Ib} / \mathrm{c}$ progenitors of $M_{R}=-4.3,-5.7,-5.7,-6.0,-6.0$, $-6.1,-6.7-6.9,-7.0$, and $-7.3 \mathrm{mag}$, we can estimate the expected number of secondary detections for any primary mass and binary model. The constraint is relatively weak, but starting to constrain the binary fraction from above. For $M_{p}=40 M_{\odot}$ and $F=1$, the expected numbers of detectable secondaries in this sample given the magnitude limits are $\sim 0.7,1.0$, and 0.5 for the low-mass, uniform, and twins distributions, respectively, corresponding to $30 \%-50 \%$ probabilities of no detections. In this case, the twins distribution has fewer detectable secondaries than the uniform distribution because many of the twins are also optically faint stripped stars (see Figure 1). The results for any of the other secondary distributions are similar-only cases like the $F=1, M_{p}=30 M_{\odot}$ twins distribution can be ruled out with high $(95 \%)$ confidence. This stronger constraint for $30 M_{\odot}$ arises from the lack of stripped secondaries at $30 M_{\odot}$ compared to $40 M_{\odot}$ seen in Figure 1 . However, a continued failure to detect "progenitors" to Type $\mathrm{Ib} / \mathrm{c}$, particularly with limits as strong as those of Crockett et al. (2007) for SN 2002ap, will begin to strongly constrain secondary distributions.

The existence of some binaries does constrain the models from below, although the limits will depend much on the definition of the problem. Consider one example. Assume there have been 22 nearby SN II for which adequate pre-supernova imaging data exists - the 20 SN from Smartt et al. (2009) plus SN 1987A and SN 1993J. In our present model, where we have no binary interactions, both of these latter SN would be normal Type II SN. Based on the binary models of SN 1993J, we compute the number of systems out of these 22 that would have a secondary within $10 \%(50 \%)$ of the mass of the primary. To have a $50 \%$ chance of at least one such system requires $F>0.73,(0.11), 0.36(0.07)$, and 0.07 (0.05) for the low-mass, uniform, and twins models, respectively. Alternatively, if we interpret SN 1987A as the SN of a secondary, then we must have $F>0.11,0.06$, and 0.04 , respectively, to have a $50 \%$ chance of having observed such an event. The constraints are again fairly weak, except that the binary fraction must be very high for the low-mass binary distribution to have any likelihood of a system like SN 1993J.

Finally, we can consider the absence of $V$-band excesses at the level of approximately $1 \mathrm{mag}$ for the Type IIP SN 2008bk and SN 2005cs, both of which have estimated progenitor masses near $M_{p} \simeq 10 M_{\odot}$. The fractions of ccSN with a secondary exceeding this limit are approximately 5\%, 9\%, and $31 \%$ for the low-mass, uniform, and twins secondary distributions and $F=1$. Thus, while two such non-detections do not constrain models, only a few additional, stronger or bluer limits are needed to begin limiting the twins distribution.

\section{IDENTIFYING AND VERIFYING ccSN SECONDARIES}

There are two additional challenges for the problem of identifying ccSN secondaries. First, if searches are only feasible for $D<10 \mathrm{Mpc}$, the statistics will be poor and grow slowly unless the search can be extended to historical ccSN where precise astrometric positions are lacking. Second, once there is a candidate secondary, we need some means of ruling out chance alignments with an unassociated star. We discuss the general problem of locating historical ccSN in the Appendix, while here we focus on the solution to both of these problems for bright ccSN secondaries.

Once the ccSN has faded, the developing SN remnant (SNR) enters the ejecta-dominated phase. Most of the ejecta is cool due to adiabatic expansion. At the surface there is a thin layer of shocked circumstellar material (CSM) and SN material separated by a contact discontinuity (see, e.g., Chevalier 1982; Chevalier \& Fransson 1994). Thus, searches for young SNR are not very successful because they search for optical (emission line), radio, or X-ray emission (see the Appendix) created by this thin sheath, and this is generally not very luminous unless the CSM is very dense.

Cool, largely neutral gas is best found by absorption, and this was dramatically demonstrated by the discovery of the remnant of the Type Ia SN 1885 in M31 by Fesen et al. (1989). SN 1885 is now easily visible as a $\sim 0$ '. 8 diameter absorption disk against the bulge of M31 at the wavelengths of high optical depth absorption lines such as Ca I (4600 ̊), Ca II H\&K, Fe I 
(3021, 3720 A), and Fe II (2300-2600 Å; Fesen et al. 2007). The same effects will be seen for ccSN. For example, if we take a representative Type II model, the $12 M_{\odot}$ S15A model from Woosley \& Weaver (1995), the $\mathrm{Na}, \mathrm{Ca}$, and Fe yields are of order $9 \times 10^{-4}, 1 \times 10^{-2}$, and $5 \times 10^{-2} M_{\odot}$, corresponding surface number densities in the remnant of order

$\Sigma \sim\{2,20,40\} \times 10^{16}\left(\frac{5000 \mathrm{~km} \mathrm{~s}^{-1}}{v}\right)^{2}\left(\frac{100 \text { years }}{t}\right)^{2} \mathrm{~cm}^{-2}$

for an expansion rate $v$ and elapsed time $t$. In full, self-similar models of the ejecta-dominated phase, the expansion of the contact discontinuity is slightly slower (radius $\propto t^{\sim 0.9}$ ) than this simple, ballistic scaling (Chevalier 1982). If we integrate over the SNR, then the material is spread over the full velocity range, so the optical depth at line center is of order

$$
\tau=\tau_{0}\left(\frac{5000 \mathrm{~km} \mathrm{~s}^{-1}}{v}\right)^{3}\left(\frac{100 \text { years }}{t}\right)^{2},
$$

where $\tau_{0} \simeq 300 x, 1100 x$, and $1800 x$ for the Na I (5890, $5895 \AA$ ), Ca I (4579-4585 ̊), and Ca II (3934, $3968 \AA$ ) lines, and $x$ is the fraction in the appropriate ionization state. The $\mathrm{Fe}$ I complex near $3800 \AA$ aill have a similar optical depth. Provided $x \gtrsim 10^{-3}$ of the material is in the ground or first excited state, as is true of the Chevalier \& Fransson (1994) models, the SNR will be optically thick to these absorption lines.

The key question is the evolution of the ionization fraction over the first millennium. For the (probably) Type Ia SN 1006, no Fe I absorption is observed, but there is modest Fe II absorption corresponding to a surface density of order $10^{15} \mathrm{~cm}^{-2}$ (Fesen et al. 1988). Models by Hamilton \& Fesen (1988) of the SN 1006 remnant found that $\mathrm{Fe} \mathrm{I}$ is largely photoionized after a few 100 years while there is still a significant fraction of Fe II. The Fe I absorption in the Tycho SNR (SN 1572) is also weak (Ihara et al. 2007), so it seems likely that the low ionization states only survive for a few hundred years in Type Ia SNRs. There is no comparable information for ccSN remnants. The Crab remnant (SN 1054) shows no strong, broad absorption lines (Sollerman et al. 2000) and we could find no results for Kepler's SN 1604 or Cassiopeia A ( $\sim 300$ years).

For the same physical size, ccSN remnants are likely to be in lower ionization states than Type Ia remnants because their higher masses and smaller expansion rates mean they have more material to photoionize and higher recombination rates. However, we must also consider the ionizing effects of the secondary (or any other central source). While a full photoionization calculation including estimates of line emission is well beyond the scope of this paper, we did examine the photoionization of a homologously expanding, homogeneous envelope of pure $\mathrm{H}$ at the level of Osterbrock (1974). We computed the timescale at which an ejecta mass of $M_{e}$ expanding at $5000 \mathrm{~km} \mathrm{~s}^{-1}$ at the outer edge of the SNR is re-ionized given an effective ionizing flux from the secondary of $Q$ photons/s, assuming case B recombination and a temperature of $10^{4} \mathrm{~K}$ for the re-ionized material. For $M_{e}=10 M_{\odot}$ and $\log Q=49$ (an O6 star roughly), the re-ionization front reached the surface of the SNR after 150 years. The re-ionization time then scales linearly with changes in $M_{e}$ and $Q$. Thus, even in the presence of a very hot, luminous secondary, there will be an extended period during which the bulk of the SNR is neutral, although a hot secondary will typically re-ionize the SNR before the reverse shock does so. For hotter stars, the re-ionization of $\mathrm{He}$ will be significantly more important than in a normal $\mathrm{H}$ II region because of its relatively higher abundance, particularly if the progenitor star was stripped prior to the explosion.

The discovery of the SN 1885 SNR in absorption against the bulge of M31 is difficult to apply to more distant SNR. First, the remnant must be resolved in order to produce an observable signal. The shell radius is approximately

$$
r_{s}=\frac{v t}{D}=0^{\prime} 035\left(\frac{v}{5000 \mathrm{~km} \mathrm{~s}^{-1}}\right)\left(\frac{t}{100 \text { years }}\right)\left(\frac{3 \mathrm{Mpc}}{D}\right),
$$

where $D$ is the distance. When the remnant is unresolved, the fractional drop in flux relative to an adjacent, unobscured wavelength is

$$
\frac{\Delta F}{F} \simeq 0.5 f_{\text {back }}\left(\frac{v}{5000 \mathrm{~km} \mathrm{~s}^{-1}}\right)^{2}\left(\frac{t}{100 \text { years }}\right)^{2}\left(\frac{3 \mathrm{Mpc}}{D}\right)^{2},
$$

where $f_{\text {back }}$ is the fraction of the local emission behind the SNR and we have assumed an $H S T$ point-spread function (PSF) radius of $r_{\mathrm{psf}}=00^{\prime} 05>r_{s}$. The signal is heavily diluted by the background light encompassed by the PSF but not behind the SNR. Once the remnant is old enough, $t \gtrsim 100(D / 3 \mathrm{Mpc})\left(v / 5000 \mathrm{~km} \mathrm{~s}^{-1}\right)$ years, to be resolved $\left(2 r_{s}>\right.$ FWHM $=0$ ' 1 ), the fractional drop of $\Delta F / F=f_{\text {back }}$ is simply the fraction of background light for an optically thick transition. Second, the change in surface brightness between a broad off-band ( $500 \AA$ ) and narrow on-band $(50 \AA)$ observation must be detectable. For filters of equal efficiency, observations using the optimal 1:3 exposure time ratio for a 10:1 bandpass ratio and assuming perfect image subtraction (off-band - onband) to produce a difference image of the absorbed flux, the noise in the difference image is four times worse than that of an image through the broad filter with the same total integration time. Thus, the signal-to-noise ratio $(\mathrm{S} / \mathrm{N})$ for a resolved $\left(r_{s}>r_{\mathrm{psf}}\right)$ absorption disk is

$$
\begin{aligned}
(\mathrm{S} / \mathrm{N})_{\mathrm{disk}} & \simeq \frac{f_{\text {back }}}{4.2}(\mathrm{~S} / \mathrm{N})_{\text {cont }}\left(\frac{r_{s}}{r_{\mathrm{psf}}}\right) \\
& \simeq f_{\text {back }}\left(\frac{r_{s}}{r_{\mathrm{psf}}}\right)\left(\frac{t_{\mathrm{exp}}}{2500 \mathrm{~s}}\right)^{1 / 2} 10^{-0.4\left(\mu_{B}-22.5\right)}
\end{aligned}
$$

scaled by the continuum signal-to-noise ratio $(\mathrm{S} / \mathrm{N})_{\text {cont }}$ for the HST WFC3/F438W filter and an exposure time of approximately one orbit. Significant detections against the unresolved emission in the disk of a galaxy are challenging - one needs old ( $r_{s}$ several times $\left.r_{\mathrm{psf}}\right)$, foreground $\left(f_{\text {back }} \simeq 1\right.$ ) systems in high surface brightness $\left(\mu_{B} \lesssim 22 \mathrm{mag} \operatorname{arcsec}^{-2}\right)$ regions of the disk.

Disks, however, are very different from bulges, because mean surface brightness has little meaning for nearby galaxies on angular scales where a significant fraction of the average surface brightness is due to individually detected stars. For a resolved star behind the remnant, the $\mathrm{S} / \mathrm{N}$ is

$$
(\mathrm{S} / \mathrm{N})_{\mathrm{disk}} \simeq \frac{1}{4.2}(\mathrm{~S} / \mathrm{N})_{\mathrm{cont}} \simeq 10\left(\frac{t_{\mathrm{exp}}}{2500 \mathrm{~s}}\right)^{1 / 2} 10^{-0.4(B-24)} .
$$

This is a far easier observation, and the probability of detection is largely set by the probability of finding a star behind the remnant. This has enormous implications when searching for secondaries to ccSN or trying to use secondaries to localize $\mathrm{ccSN}$ because the secondaries are always inside the remnant. 
Moreover, as pointed out by Ozaki \& Shigeyama (2006), in the context of searches for binary companions to the progenitor of the Type Ia Tycho SN, their absorption signature is unique. Foreground stars show no absorption, background stars show both redshifted and blueshifted absorption, and companions show only blueshifted absorption. This means that searches for bright ( $\gtrsim 26 \mathrm{mag}$ ) companions can be carried out in finite observing time $(\lesssim 10$ orbits) for the hundreds of years between when the direct emission from the SN/SNR ceases and when the remnant re-ionizes.

\section{DISCUSSION}

The current picture of massive stars and the creation of ccSN implies that a large fraction $(\gtrsim 50 \%)$ of SN progenitors are in stellar binaries at the time of the explosion. In many cases, the secondary stars are detectable in either pre-explosion or latetime observations. In pre-explosion images, the secondary to a Type II SN is most observable as a blue excess in the progenitor SED with $10 \%$ excesses being relatively common $(25 \%-50 \%)$ and $100 \%$ excesses being relatively rare $(10 \%-25 \%)$. Excesses become more common as the secondary mass distribution is skewed to higher masses. The secondary to a Type Ib/c SN will frequently $(\sim 50 \%)$ be the more optically luminous star, because the stripped primary is radiating primarily in the ultraviolet. Direct observations of secondaries are, however, challenging, and are most feasible for SN closer than $\sim 10 \mathrm{Mpc}$.

There are, at present, no systematic attempts to survey for secondaries other than Ryder et al.'s (2006) deliberate search for a secondary star to the Type IIb SN 2001ig. The data that are available permit a binary fraction $F=1$, although the limits on Type Ib/c progenitors (Smartt 2009) and on color excesses for two Type II progenitors (Maund et al. 2005; Li et al. 2006; Mattila et al. 2008) begin to weakly constrain $F \simeq 1$ for a "twins" distribution of secondaries where $50 \%$ of secondaries have $90 \%-100 \%$ the mass of the primary. As progenitor statistics improve due to the accumulation of both $\mathrm{SN}$ and adequate archival data, the data will begin to constrain these models. It is worth, however, considering the types of observations needed to constrain the secondary population.

In pre-explosion images, the challenge is to recognize that the SED is a blend of two stars, as Aldering et al. (1994) found for SN 1993J. For the Type Ib/c SN, this may be difficult because both stars will typically be blue, but for Type II SN the signature will generally be a blue excess to a red star. To date, most detections of SN progenitors are at best in two bands, allowing the determination of a luminosity and either one intrinsic color or an extinction (see Smartt et al. 2009). Limiting the presence of a secondary will generally require at least three bands. Ideally, a complete three-band HST survey of the $\sim 40$ nearby $(\lesssim 10 \mathrm{Mpc})$ galaxies that dominate the local supernova rate $(\simeq 1$ /year; see Kochanek et al. 2008) would provide an archival legacy for finding both progenitors and secondaries into the future as well as for a broad range of other astrophysical studies. We should note, however, that the recent transients in NGC 300 and SN 2008S in NGC 6946, whatever their underlying mechanism, show that mid-IR observations are also crucial to understanding progenitor systems because in some cases they are self-obscured by dust (see Prieto et al. 2008b and related references).

The most dramatic possibility in pre-explosion observations is the identification of an eclipsing binary as the progenitor system. Very little is known about the time variability of progenitor systems, with upper bounds $\sim 0.2-0.3$ mag for both SN 1987A over its last century (see Plotkin \& Clayton 2004, and references therein) and for SN 1993J over a 150 day period in 1984 (Cohen et al. 1995). Few local galaxies have been the subject of the relatively deep, long-duration monitoring projects that would be needed to detect eclipses in these (super)giant stars. Discovery of even a single example would have dramatic physical consequences, not only by making a direct link between binary evolution and a resulting supernova, but because of the enormous amount of extra physical information that can be derived from the binary period and eclipse properties. These will, of course, be rare, but we note that for the parameters of the Maund et al. (2004) model of SN 1993J, the probability of it having been an eclipsing binary exceeds $10 \%$, with eclipse durations of roughly two years occurring every 10 years. While challenging, the necessary data can be obtained by ground-based monitoring of nearby galaxies, as illustrated by the eclipsing binary in Holmberg IX, a tidal dwarf companion to M81, discovered by Prieto et al. (2008a) using the Large Binocular Telescope. In addition to monitoring any $\mathrm{SN}$ progenitors, such data would also inventory all luminous eclipsing binaries and variable stars (e.g., Cepheids) as well as providing the basis for determining whether any massive stars die as failed supernovae without a dramatic explosion (Kochanek et al. 2008). A single epoch, higher resolution HST survey is an important complement to any ground-based monitoring. While difference imaging methods make it relatively easy to obtain light curves of variable sources from the ground even at $10 \mathrm{Mpc}$ (the AC signal), stellar crowding means that HST is needed to provide absolute calibrations (the DC signal).

Once the SN has faded, it is relatively easy to find and confirm bright secondary stars of ccSN in galaxies closer than $D<10 \mathrm{Mpc}$. During its coasting phase, the SNR will have very high absorption optical depths in lines such as Ca I, Ca II, Na I, Fe I, and Fe II, as Fesen et al. (1989, 2007) have already used to identify the SNR of the Type Ia SN 1885 in M31, unless something photoionizes the gas. The method used for SN 1885 , searching for a disk of absorption against the emission from unresolved background stars, is possible only when the surface brightness is relatively high ( $\left.\gtrsim 22 \mathrm{mag} \operatorname{arcsec}^{-2}\right)$ and after $\sim 10^{2}$ years when $H S T$ can resolve the SNR. This approach may work best for identifying historical ccSN associated with star clusters (e.g., like SN 2004am and SN 2004dj in the Smartt et al. 2009 sample). On the other hand, any resolved star brighter than $\lesssim 26$ mag that is either behind or inside the SNR should produce a detectable absorption signal. The two cases can be distinguished because background stars will show both redshifted and blueshifted absorption, while secondaries will show only blueshifted absorption (Ozaki \& Shigeyama 2006). Moreover, if the search is carried out shortly after the SN fades, contamination by a background star is very unlikely given the compactness (milliarcseconds after 10 years) of the SNR. Fortunately, ccSN secondaries are generally blue stars at the wavelengths likely to show strong absorption features, although some care will be needed for stars with significant Balmer absorption in their atmospheres.

Existing studies of re-ionization of SNR by either emission from shocked material near the surface or ambient UV backgrounds (e.g., Chevalier 1982; Hamilton \& Fesen 1988; Chevalier \& Fransson 1994; Fesen et al. 2007) suggest that the bulk of an SNR should remain neutral for several centuries but then re-ionize on timescales $\lesssim 10^{3}$ years, although the late-time analyses of this problem have all focused on Type Ia remnants. These timescales seem to be broadly consistent with the limited available data on absorption in SNR. The effect of 
secondary stars on the re-ionization of remnants seems never to have been considered. Crude models suggest that hot, luminous O star secondaries can photoionize an SNR from the inside on timescales of $10^{2}-10^{3}$ years. This will create a very peculiar cross between an $\mathrm{H}$ iI region with the secondary star as the photoionizing source, a planetary nebula, because of the very high $\mathrm{He}$ and metal abundances, and an SNR because of the high ejecta velocities. Full, dynamical, radiative transfer simulations will be needed to characterize the observability of this phase. The obvious trial scenario, the late-time spectra of SN 1993J (e.g., Maund \& Smartt 2009), is probably little affected by this process because the secondary is a cooler B star.

The first step in a campaign to identify secondaries is to take all nearby ccSN with accurate positions, particularly from post-explosion $H S T$ imaging, and re-examine the sites. Ideally, one would start with systems for which late-time observations already exist (e.g., the $11 \mathrm{ccSN}$ examined by Li et al. (2002), although these are generally more distant than optimal) to ensure that any source is unlikely to be originating from the SN/SNR. The next step is to use the absorption method to try to confirm some of the candidates. Here the obvious first cases to try are the candidate secondary to SN 2001ig identified by Ryder et al. (2006) and, once the SN has faded enough to examine absorption in the secondary without contamination from emission by the SNR, the secondary star of SN 1993J and (possibly) SN 2008ax. The numbers and properties of the secondary population will constrain the role of binaries in the evolution of massive stars and the production of the various classes of ccSN. They also provide an independent check of the models used to infer the properties of progenitors. In a few cases where the secondary is sufficiently bright, it may be possible to examine abundances in the ccSN ejecta. In the early phases, even elements with ejecta masses of $\sim 10^{-6} M_{\odot}$ (e.g., Barium) have significant absorption optical depths, although detection may be limited by Doppler smearing with stronger lines and inferences may be limited by ejecta clumping. Identifying the secondary to a historical SN will also establish that the SN was not a Type Ia and set a lower bound to the mass of the primary.

I thank J. Beacom, R. Chevalier, A. Hamilton, J. Johnson, M. Pinsonneault, R. Pogge, J. Prieto, S. Smartt, K. Stanek, and T. Thompson for comments and discussion. This research made use of the Sternberg Astronomical Institute supernova catalogs and the NASA/IPAC Extragalactic Database (NED), which is operated by JPL/Caltech, under contract with NASA. This research was supported by NSF grant AST-0908816.

\section{APPENDIX}

\section{OTHER APPROACHES TO IDENTIFYING HISTORICAL SUPERNOVAE}

In Section 5, we introduced a simple means of identifying bright secondaries to ccSN even if the ccSN location is poorly known. This method should work for several hundred years after the SN unless something photoionizes the remnant. The absorption method will also work if the SNR lies in front of a sufficiently bright background star or if it lies in a region of high surface brightness like a star cluster. Here we discuss other possibilities for identifying the positions of historical ccSN with high precision (Smartt et al. (2009) argue for a goal of order 10 mas, albeit at higher typical distances than $10 \mathrm{Mpc}$ ). To provide a sense of the problem, Barth et al. (1996) and Van Dyk et al. (1999) examined the environments of past
SN with HST imaging data, but could only localize the SN with positional uncertainties of 1-10 arcsec that made anything beyond environmental studies impossible.

The goal of most searches for SNR in external galaxies has been to carry out more uniform surveys than are possible in the Galaxy. Searches have used optical emission lines (e.g., Matonick \& Fesen 1997), radio emission (e.g., Lacey et al. 1997), and X-ray emission (e.g., Pannuti et al. 2007). The optical and radio surveys do not find counterparts to historical supernovae unless they had strong CSM interactions such as the examples we discuss below. Specific X-ray searches have been more successful, with X-ray counterparts having been identified for most post-1970 SN ${ }^{3}$ with the record being SN 1941C (Soria $\&$ Perna 2008). In general, there is little overlap between the sources found by the three approaches (see, e.g., Pannuti et al. 2007). A further problem is that radio and X-ray detections may not lead to good enough optical astrometry in these crowded fields due to problems in matching the reference frames with high accuracy.

Two additional possibilities are late-time light curves and dust echoes. The SN themselves fade relatively rapidly. For example, SN 1987A was fainter than its progenitor by $\sim 800$ days (Suntzeff et al. 1991). Li et al. (2002) observed $11 \mathrm{ccSN}$ (mostly Type IIn) at late times. The four observed later than 1000 days had detections interpreted to be the SN but spanning an interesting range for secondaries $\left(M_{V} \sim-4\right.$ to $-13 \mathrm{mag}$, see Figure 2). Extrapolating the light curves using the mean decline rates suggests that in most cases the $\mathrm{SNe}$ are fainter than $M_{V} \sim$ -4 mag after 2000 days. On these long timescales, the only internal source of energy is ${ }^{44} \mathrm{Ti}$, which produces a maximum luminosity of $L_{44} \simeq 10^{3}\left(M_{44} / 10^{-4} M_{\odot}\right) \exp (-t / 86$ years $) L_{\odot}$ if all the decay energy is absorbed and $25 \%$ of this if only the positrons are absorbed (Woosley et al. 1989). Combined

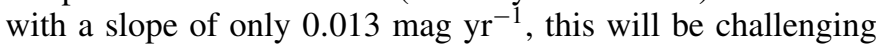
to detect decades later even if the emission is concentrated in the optical/near-IR. Some older ccSN are, however, trivial to find decades after the event because of CSM interactions. In particular, bright radio supernovae, such as SN 1979C (Milisavljevic et al. 2009), SN 1980K (Fesen et al. 1999), SN 1986J (Milisavljevic et al. 2008), or SN 1993J (Maund et al. 2004), are still being observed. The general distribution of supernovae from the nearly invisible ${ }^{44} \mathrm{Ti}$ regime to the strong CSM interaction regime is not known. One result of searching for secondaries to all ccSN with accurate post-explosion images, would be to clarify these issues and provide strategies for the $\mathrm{ccSN}$ without such data.

The last possibility we consider is searching for dust echoes. Extragalactic dust echoes have been imaged for the Type IIpec SN 1987A (Crotts 1988), the Type Ia SN 1991T (Sparks et al. 1999), the Type IIb SN 1993J (Sugerman \& Crotts 2002; Liu et al. 2003), the Type IIP SN2003gd (Sugerman 2005), the Type Ia SN 1998bu (Cappellaro et al. 2001), the Type Ia SN 1995E (Quinn et al. 2006), and the Type Ia SN 2006X (Wang et al. 2008). They were generally found using difference imaging techniques, taking advantage of the frequently superluminal pattern speeds of the echoes. While these detections are all relatively close in time to the SN, Rest et al. (2005) have demonstrated that echoes of 400-600 year old supernovae can be seen in the LMC, some of which also have superluminal apparent motions (up to $v_{e}=3 c$ ). The LMC echoes are resolved ( $\Delta \simeq 2$ '.5 across) and can have relatively high surface

\footnotetext{
See http://lheawww.gsfc.nasa.gov/users/immler/supernovae_list.html.
} 
brightnesses (up to $22 \mathrm{mag} \operatorname{arcsec}^{-2}$ ). There have also been ground-based attempts to find dust echoes in more distant galaxies. Boffi et al. (1999) examined the sites of historical SN for unusually blue emission due to scattered light, finding 16 candidates among 64 nearby SN, and Romaniello et al. (2005) searched for polarized emission near four SN in M 83 without success.

The problem with any search for dust echoes is recognizing them against the crowded stellar background. The successful detections have all taken advantage of the apparent motions of echoes and difference imaging multiple epochs to solve this problem. Here we note that observations comparable to Rest et al. (2005) are possible for older SN in nearby galaxies using $H S T$, particularly if there is dust in the foreground of the SN. The outer angular scale of the echoes is determined by the elapsed time and the foreground dust distribution, with

$$
\begin{aligned}
r_{\text {out }}= & 0.54\left(\frac{t}{10 \text { years }}\right)^{1 / 2}\left|\frac{z_{\text {dust }}}{10 \mathrm{pc}}\right|^{1 / 2} \\
& \times\left(\frac{3 \mathrm{Mpc}}{D}\right)\left|1+\frac{c t}{2 z_{\text {dust }}}\right|^{1 / 2},
\end{aligned}
$$

where $z_{\text {dust }}$ is the distance of the dust along the line of sight from the SN. Thus, the angular scales for nearby $(D<10 \mathrm{Mpc})$ somewhat old (decades to centuries) ccSN dust echoes are well suited to HST. Theoretical models of echoes have been explored by Sugerman (2003) and Patat (2005), but we will focus on scaling from the observed properties of the many century old LMC echoes. The surface brightnesses of the brighter LMC echoes can be very high, but $H S T$ will somewhat under resolve them in one dimension because their widths $\left(\Delta \sim 0^{\prime \prime}\right.$.04(3 Mpc/D)) will be somewhat narrower than HST's PSF. Failure to resolve the echo in one dimension reduces its effective surface brightness as $2.5 \log \Delta / \mathrm{FWHM}$, where $\mathrm{FWHM} \simeq 0^{\prime \prime} 1$ is the resolution of $H S T$. Even so, high surface brightness echoes at $D<10 \mathrm{Mpc}$ should be relatively easy to detect if the stellar backgrounds can be suppressed. The existing $H S T$ studies have all used $H S T$ pre-explosion data to subtract the stellar background and render the echoes easily visible. This is not feasible for historical SN, but, the strategy used for the LMC detections will work. The proper motion of an echo with an effective velocity $v_{e}$ is

$$
\mu=0.021\left(\frac{v_{e}}{c}\right)\left(\frac{3 \mathrm{Mpc}}{D}\right) \text { year }^{-1}
$$

and it must move one resolution element, $\mu \Delta t=$ FWHM for difference imaging to be used. The required timescale is relatively short

$$
\Delta t \simeq 5\left(\frac{c}{v_{e}}\right)\left(\frac{D}{3 \mathrm{Mpc}}\right) \text { years }
$$

even in the absence of superluminal apparent velocities. This can be tested for many $\mathrm{SNe}$ as part of any late-time search for secondary stars or by revisiting the SN studied by Barth et al. (1996), Van Dyk et al. (1999), and Li et al. (2002).

\section{REFERENCES}

Aldering, G., Humphreys, R. M., \& Richmond, M. 1994, AJ, 107, 662 Anderson, J. P., \& James, P. A. 2008, MNRAS, 390, 1527

Barth, A. J., van Dyk, S. D., Filippenko, A. V., Leibundgut, B., \& Richmond, M. W. 1996, AJ, 111, 2047

Belczynski, K., Kalogera, V., Rasio, F. A., Taam, R. E., Zezas, A., Bulik, T., Maccarone, T. J., \& Ivanova, N. 2008, ApJS, 174, 223
Boffi, F. R., Sparks, W. B., \& Macchetto, F. D. 1999, A\&AS, 138, 253

Cappellaro, E., et al. 2001, ApJ, 549, L215

Chevalier, R. A. 1982, ApJ, 258, 790

Chevalier, R. A., \& Fransson, C. 1994, ApJ, 420, 268

Cohen, J. G., Darling, J., \& Porter, A. 1995, AJ, 110, 308

Crockett, R. M., et al. 2007, MNRAS, 381, 835

Crockett, R. M., et al. 2008, MNRAS, 391, L5

Crotts, A. P. S. 1988, ApJ, 333, L51

de Loore, C., \& Vanbeveren, D. 1992, A\&A, 260, 273

Eldridge, J. J., Izzard, R. G., \& Tout, C. A. 2008, MNRAS, 384, 1109

Fesen, R. A., Höflich, P. A., Hamilton, A. J. S., Hammell, M. C., Gerardy, C. L., Khokhlov, A. M., \& Wheeler, J. C. 2007, ApJ, 658, 396

Fesen, R. A., Pavlov, G. G., \& Sanwal, D. 2006, ApJ, 636, 848

Fesen, R. A., Saken, J. M., \& Hamilton, A. J. S. 1989, ApJ, 341, L55

Fesen, R. A., Wu, C.-C., Leventhal, M., \& Hamilton, A. J. S. 1988, ApJ, 327 164

Fesen, R. A., et al. 1999, AJ, 117, 725

Gal-Yam, A., et al. 2007, ApJ, 656, 372

Gilmozzi, R., et al. 1987, Nature, 328, 318

Graves, G. J. M., et al. 2005, ApJ, 629, 944

Hamilton, A. J. S., \& Fesen, R. A. 1988, ApJ, 327, 178

Ihara, Y., Ozaki, J., Doi, M., Shigeyama, T., Kashikawa, N., Komiyama, K., \& Hattori, T. 2007, PASJ, 59, 811

Kobulnicky, H. A., \& Fryer, C. L. 2007, ApJ, 670, 747

Kochanek, C. S., Beacom, J. F., Kistler, M. D., Prieto, J. L., Stanek, K. Z., Thompson, T. A., \& Yuksel, H. 2008, ApJ, 684, 1336

Krause, O., Birkmann, S. M., Usuda, T., Hattori, T., Goto, M., Rieke, G. H., \& Misselt, K. A. 2008, Science, 320, 1195

Lacey, C., Duric, N., \& Goss, W. M. 1997, ApJS, 109, 417

Lejeune, T., \& Schaerer, D. 2001, A\&A, 366, 538

Levesque, E. M., Massey, P., Olsen, K. A. G., Plez, B., Josselin, E., Maeder, A., \& Meynet, G. 2005, ApJ, 628, 973

Li, W., Filippenko, A. V., Van Dyk, S. D., Hu, J., Qiu, Y., Modjaz, M., \& Leonard, D. C. 2002, PASP, 114, 403

Li, W., Van Dyk, S. D., Filippenko, A. V., Cuillandre, J.-C., Jha, S., Bloom, J. S., Riess, A. G., \& Livio, M. 2006, ApJ, 641, 1060

Li, W., Wang, X., Van Dyk, S. D., Cuillandre, J.-C., Foley, R. J., \& Filippenko, A. V. 2007, ApJ, 661, 1013

Liu, J.-F., Bregman, J. N., \& Seitzer, P. 2003, ApJ, 582, 919

Marigo, P., Girardi, L., Bressan, A., Groenewegen, M. A. T., Silva, L., \& Granato, G. L. 2008, A\&A, 482, 883

Matonick, D. M., \& Fesen, R. A. 1997, ApJS, 112, 49

Mattila, S., Smartt, S. J., Eldridge, J. J., Maund, J. R., Crockett, R. M., \& Danziger, I. J. 2008, ApJ, 688, L91

Maund, J. R., \& Smartt, S. J. 2009, Science, 324, 486

Maund, J. R., Smartt, S. J., \& Danziger, I. J. 2005, MNRAS, 364, L33

Maund, J. R., Smartt, S. J., Kudritzki, R. P., Podsiadlowski, P., \& Gilmore, G F. 2004, Nature, 427, 129

Milisavljevic, D., Fesen, R. A., Kirshner, R. P., \& Challis, P. 2009, ApJ, 692 , 839

Milisavljevic, D., Fesen, R. A., Leibundgut, B., \& Kirshner, R. P. 2008, ApJ, 684,1170

Osterbrock, D. E. 1974, Astrophysics of Gaseous Nebulae (San Francisco: Freeman)

Ozaki, J., \& Shigeyama, T. 2006, ApJ, 644, 954

Pannuti, T. G., Schlegel, E. M., \& Lacey, C. K. 2007, AJ, 133, 1361

Patat, F. 2005, MNRAS, 357, 1161

Pinsonneault, M. H., \& Stanek, K. Z. 2006, ApJ, 639, L67

Plotkin, R. M., \& Clayton, G. C. 2004, J. Am. Assoc. Var. Star Obs., 32, 89

Podsiadlowski, P., Hsu, J. J. L., Joss, P. C., \& Ross, R. R. 1993, Nature, 364, 509

Podsiadlowski, P., \& Joss, P. C. 1989, Nature, 338, 401

Podsiadlowski, P., Joss, P. C., \& Hsu, J. J. L. 1992, ApJ, 391, 246

Prieto, J. L., et al. 2008a, ApJ, 673, L59

Prieto, J. L., et al. 2008b, ApJ, 681, L9

Quinn, J. L., Garnavich, P. M., Li, W., Panagia, N., Riess, A., Schmidt, B. P., \& Della Valle, M. 2006, ApJ, 652, 512

Rest, A., et al. 2005, Nature, 438, 1132

Romaniello, M., Patat, F., Panagia, N., Sparks, W. B., Gilmozzi, R., \& Spyromilio, J. 2005, ApJ, 629, 250

Ryder, S. D., Murrowood, C. E., \& Stathakis, R. A. 2006, MNRAS, 369, L32

Smartt, S. J. 2009, ARA\&A, 47, 63

Smartt, S. J., Eldridge, J. J., Crockett, R. M., \& Maund, J. R. 2009, MNRAS, 395, 1409

Sollerman, J., Lundqvist, P., Lindler, D., Chevalier, R. A., Fransson, C., Gull, T. R., Pun, C. S. J., \& Sonneborn, G. 2000, ApJ, 537, 861

Soria, R., \& Perna, R. 2008, ApJ, 683, 767 
Sparks, W. B., Macchetto, F., Panagia, N., Boffi, F. R., Branch, D., Hazen, M. L., \& della Valle, M. 1999, ApJ, 523, 585

Stancliffe, R. J., \& Eldridge, J. J. 2009, MNRAS, 396, 1699

Sugerman, B. E. K. 2003, AJ, 126, 1939

Sugerman, B. E. K. 2005, ApJ, 632, L17

Sugerman, B. E. K., \& Crotts, A. P. S. 2002, ApJ, 581, L97

Suntzeff, N. B., Phillips, M. M., Depoy, D. L., Elias, J. H., \& Walker, A. R. 1991, AJ, 102, 1118
Van Dyk, S. D. 2004, New Astron. Rev., 48, 749

Van Dyk, S. D., Peng, C. Y., Barth, A. J., \& Filippenko, A. V. 1999, AJ, 118, 2331

Wang, X., Li, W., Filippenko, A. V., Foley, R. J., Smith, N., \& Wang, L. 2008, ApJ, 677, 1060

Woosley, S. E., Hartmann, D., \& Pinto, P. A. 1989, ApJ, 346, 395

Woosley, S. E., \& Weaver, T. A. 1995, ApJS, 101, 181

Zinnecker, H., \& Yorke, H. W. 2007, ARA\&A, 45, 481 